Review

\title{
Salmonella as an Innovative Therapeutic Antitumor Agent
}

\author{
Wen-Wei Chang ${ }^{1,2}$ and Che-Hsin Lee ${ }^{3, *}$
}

1 Department of Biomedical Sciences, College of Medical Science and Technology, Chung Shan Medical University, Taichung 402, Taiwan; E-Mail: changww@csmu.edu.tw Department of Medical Research, Chung Shan Medical University Hospital, Taichung 402, Taiwan

3 Department of Microbiology, School of Medicine, China Medical University, Taichung 404, Taiwan

* Author to whom correspondence should be addressed; E-Mail: chlee@mail.cmu.edu.tw; Tel.: +886-4-2205-3366 (ext. 2173); Fax: +886-4-2205-3764.

Received: 24 June 2014; in revised form: 28 July 2014 / Accepted: 18 August 2014 /

Published: 21 August 2014

\begin{abstract}
Lack of specificity of the therapeutic agent is a primary limitation in the treatment of a tumor. The use of preferentially replicating bacteria as therapeutic agents is an innovative approach to tumor treatment. This is based on the observation that certain obligate or facultative anaerobic bacteria are capable of multiplying selectively in tumors and inhibiting their growth. Bacteria have been employed as antitumor agents that are capable of preferentially amplifying within tumors and inhibiting their growth. Moreover, bacteria-derived factors have an immune-stimulation effect. Therefore, bacteria are able to transfer therapeutic genes into the tumor cells using their infective ability. Herein, we introduce the application of bacteria for tumor therapy and focus on Salmonella, which have been widely used for tumor therapy. Salmonella have mainly been applied as gene-delivery vectors, antitumor immune activators and tumor cell death inducers. This study will not only evaluate the therapeutic efficacy of Salmonella for the treatment of tumor but will also elucidate the mechanisms underlying the antitumor activities mediated by Salmonella, which involve host immune responses and cellular molecular responses.
\end{abstract}

Keywords: Salmonella; tumor-targeting; antitumor; immunotherapy 


\section{Introduction}

Recently, general tumor treatments including surgery, radiation therapy, and chemotherapy have shown some limitations. Surgery and radiation therapy are limited to localized tumors [1], and chemotherapy may induce severe side effects. Salmonella are gram-negative, facultative anaerobes that are a common cause of intestinal infections. Due to substantial immunostimulation produced by Salmonella lipopolysaccharide (LPS) and other components, systemic infection with Salmonella induces proinflammatory cytokine expression and immune cell infiltration in the host [2]. Systemically administered Salmonella is preferentially accumulated within tumors for at least one month, forming tumor-to-normal-tissue ratios exceeding 1000-10,000 to 1 [3]. Furthermore, this accumulation is accompanied by a delay in the growth of the tumor [4,5]. Salmonella can grow under both aerobic and anaerobic conditions, so they are able to colonize small metastatic tumors as well as larger tumors [6-8]. Attenuated Salmonella hinder tumor growth in a broad range of human and mouse tumors $[9,10]$. Tumor growth is inhibited for long periods, even up to several weeks. These observations, coupled with ease of genetic manipulation, suggest that Salmonella are good candidates for therapeutic antitumor agents, and genetically engineered Salmonella have been developed to express exogenous genes, aiming to enhance their antitumor effects $[2,5,11]$.

\section{Host Immunity and Salmonella}

\subsection{Innate Immunity and Salmonella}

The immune response against Salmonella is composed of an immediate response mediated by the innate arm of the immune system followed by antigen-specific adaptive immunity [12]. Together, these two arms of the immune system work to eradicate infection and provide long-lasting immunity and memory [13]. Salmonella-induced tumor inhibition occurs with alterations in myeloid cells consistent with maturation to macrophage effectors and a reduction in their suppressive capacity. The antitumor effectiveness of Salmonella relies on the induction of the innate immune response through the toll-like receptor-myeloid differentiation primary response gene (TLR-MYD88) signaling pathway [14]. This is consistent with our previous report demonstrating that Salmonella induce cytokine production and antitumor activities via TLR4 signaling, which may help clarify the molecular mechanism of Salmonella-induced host antitumor responses [15]. The increased expression of interferon (IFN)-induced chemokines in the tumor was observed during Salmonella treatment in vivo. IFN-dependent chemokines induced by Salmonella, such as monokine induced by IFN- $\gamma$ (MIC) and IFN-inducible protein-10 (IP-10), are expected to recruit activated effector cells within the tumor. Taken together, we and other groups show that Salmonella significantly upregulates IFN- $\gamma$ [15-17] and the IFN-inducible chemokines MIG and IP-10, which may be responsible for recruiting peripheral natural killer and $\mathrm{T}$ cells to the tumor [15].

\subsection{T Cell Activation by Salmonella}

Salmonella-induced immune responses, especially $\mathrm{T}$ cell activation by Salmonella, may include both anti-Salmonella-specific and tumor antigen-specific [18,19]. Rescigno et al. demonstrated that 
bacterial components, such as lipopolysaccharide (LPS), lipoteichoic acid (LTA), and flagellin, induced the expression of connexin 43 (Cx43) in tumor cells. Salmonella can cause melanoma cells to form gap junctions (Cx43) with adjunct immune dendritic cells. Consequently, the dendritic cells use peptides transferred from the tumor cells to stimulate $\mathrm{T}$ cells to recognize and kill the tumor cells at the primary site and prevent metastasis formation [19]. Salmonella act both locally, by recruiting T cells that inhibit tumor growth, and systemically, where Salmonella promote development of the immune response via the cross-presentation of tumor antigen [19]. Salmonella replication and lysis of tumor cells may induce cell-mediated immune responses to tumor cells by increased infiltration of $\mathrm{CD} 8^{+}$ $\mathrm{T}$ cells in Salmonella-treated tumors [20]. The cytotoxic T cell response against tumor cells may enhance the antitumor efficacy of Salmonella-expressing cytokines, exhibiting an ability to modulate host immunity and inhibit tumor growth [11]. In contrast, reducing immunosuppressive cytokines in tumor can also inhibit tumor growth [21]. Binder et al., reported on tumor-specific antigen delivery into tumors using Salmonella to increase antigen levels and generate a proinflammatory tumor microenvironment [22]. Salmonella is a powerful therapeutic approach to rescue dysfunctional endogenous tumor-specific $\mathrm{CD}^{+} \mathrm{T}$ cells. We used wild-type, $\mathrm{CD} 4^{+} \mathrm{T}$ cell-deficient and $\mathrm{CD} 8^{+} \mathrm{T}$ cell-deficient mice to study the role of $\mathrm{T}$ cells in the antitumor immune responses induced by Salmonella. When systemically administered into mice bearing tumors, Salmonella significantly inhibited tumor growth by 50\%. In contrast, in $\mathrm{CD}^{+} \mathrm{T}$ cell-deficient mice, there was only a 34\%-42\% inhibition of tumor growth [12]. Anti-Salmonella-specific $\mathrm{T}$ cells that are induced during the infection are recruited in the flamed tumor site where they kill infected tumor cells [20]. Salmonella actively infects tumor cells in the presence of an expanded pool of anti-Salmonella-specific immune responses. During the clearances of Salmonella-killed tumor cells, tumor debris could be generated in large quantities, be taken up by antigen-presenting cells including macrophage and dendritic cell, and presented to naïve $\mathrm{T}$ cells for stimulation of tumor-specific T cells. These results suggest that tumor-targeted therapy using Salmonella, which exerts tumoricidal effects and stimulates $\mathrm{T}$ cell activities, represents a potential strategy for the treatment of tumors (Figure 1).

Figure 1. Salmonella-mediated stimulation of antitumor immunity. Salmonella-activated dendritic cell or macrophage by bacterial components, such as lipopolysaccharide (LPS), lipoteichoic acid (LTA), and flagellin. Salmonella inhibits tumors by increasing infiltrating cells or by cytokine expression.

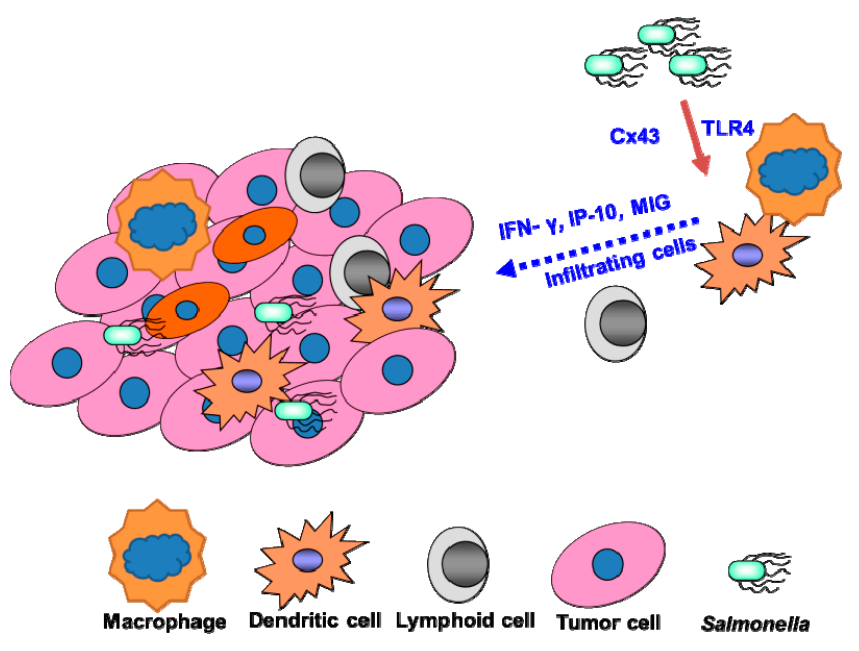




\subsection{Antibodies and Salmonella}

B cells play an important role in the antitumor activity mediated by Salmonella. Although Salmonella accumulated within the tumors in B cell-deficient mice, the bacterial loads of healthy organs were higher than those in wild-type mice. The inflammation cytokines and bacteremia were found in B cell-deficient mice after Salmonella treatment. When Salmonella accumulated within the tumor, B cells inhibited the dissemination of Salmonella to other healthy organs [23]. A major source of natural antibodies seems to be the B-1 cell subset. There is also evidence for natural antibodies with specificities for a wide range of bacterial antigens [24]. The natural antibodies produced by B cells take part in the control of Salmonella dissemination in tumor-bearing mice. The natural antibodies produced by B cells result in a slightly lower total number of bacteria in the tumor sites, but decreased inflammation and cytokine production in the healthy organs after systemic Salmonella treatment. A phase I study of attenuated Salmonella (VNP20009) in humans with melanoma was reported. No antitumor responses were observed and VNP20009 was detected in tumor sites in only $12.5 \%$ of patients [25]. This clinical study was not successful, which prompts the question of how these factors in the host influence the accumulation of Salmonella within the solid tumor. Salmonella have great potential as immune stimulants. However, following Salmonella administration, strong host immunity may develop. In addition, antibodies directed against Salmonella are also synthesized. The anti-Salmonella immune responses that exist in the host influence the tumor-targeting potential of Salmonella after systemic administration [13]. The loss of accumulation of Salmonella within the tumor contributes to the inhibition of the Salmonella-mediated antitumor response. These results may explain the limited accumulation of Salmonella within tumor sites and the non-significant antitumor response after systemic administration in clinical trials [13].

\section{Direct Tumor-Killing Activity of Salmonella}

Salmonella have been reported to possess antitumor activities. The mechanisms underlying their direct antitumor activities are largely unknown. Previous studies demonstrated that the induction of tumor apoptosis was correlated with Salmonella accumulation in the tumor sites [26]. Salmonella in the tumor induced apoptosis by multiple mechanisms, including competition for nutrients and stimulation of immune response [2]. Moreover, toxins from bacteria may induce the apoptosis of a tumor. As bacterial replication in tumors and subsequent lysis of tumor cells may induce cell-mediated immune responses to tumor cells, higher oncolysis could account, in part, for an increased infiltration of immune cells in tumors. The cells undergoing Salmonella-induced cell death exhibit heterogeneous morphological features [27]. It is clear that more than one mechanism is involved in the Salmonella-induced killing of cells [28]. Recently, we demonstrated that Salmonella may induce cell death via apoptosis and autophagic pathways by using an autophagy inhibitor (3-methyladenine) and an apoptosis inhibitor (Z-VAD-FMK). Autophagy is involved in the cell-defense elimination of bacteria [29]. The signaling pathways leading to activation of bacteria-induced autophagy in tumor cells remain to be elucidated. The AKT/mTOR/p70S6K signaling pathway negatively regulates autophagy. We determined that the levels of phosphorylated AKT, mTOR, and p70S6K were significantly decreased in Salmonella-treated tumor cells [30]. These results showed that Salmonella can induce autophagic activities in addition to 
caspase-dependent cell death in tumor cells (Figure 2). Autophagy may co-occur with apoptosis in tumor cells exposed to Salmonella. Furthermore, at later stages of infection, autophagy may partially participate in the execution of tumor cell death by enhancing apoptosis. When apoptosis is blocked, infected tumor cells undergo increased autophagy. These data suggest that Salmonella treatment efficiently induces both autophagy and apoptosis, which partner to induce cell death cooperatively by modifying beclin-1 and caspase expression. Apoptosis and autophagy existed crosstalk between two pathways. Salmonella induced both apoptosis and autophagy. Both apoptosis and autophagy cooperate to lead to tumor cell death after Salmonella infection.

Figure 2. Salmonella-mediated cell death pathway in tumor cells. The arrow indicates that Salmonella induces signaling pathway. The flat indicates that Salmonella inhibits signaling pathway. Salmonella induces tumor cell death by activating caspase or down-regulating the $\mathrm{AKT} / \mathrm{mTOR}$ signaling pathway.

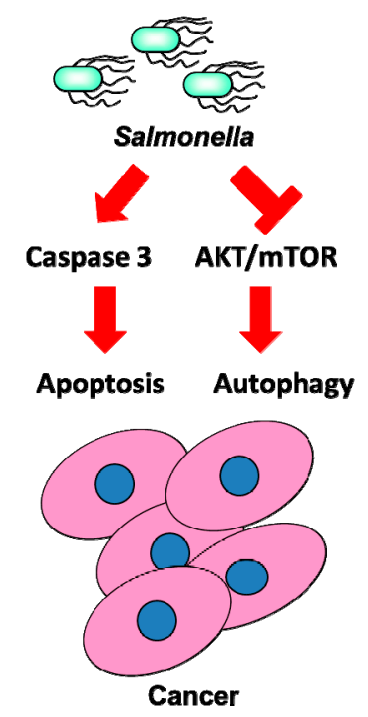

\section{Combination Therapy with Salmonella}

Avoiding side effects has emerged as a key issue in the development of safe Salmonella therapy approaches. Weiss et al., suggested that Salmonella can be readily controlled by systemic administration of antibiotics [31]. Recently, the combination of a traditional Chinese medicine herbal mixture and Salmonella enabled the bacteria to be safely administered at a high dose with increased treatment efficacy and reduced toxicity compared to monotherapy with Salmonella [32]. These results will contribute to increasing the safety and the therapeutic potential of tumor-targeting Salmonella. Previously, we exploited Salmonella as a single tumor-targeting antitumor agent and as part of a combination therapy with chemotherapy for mice bearing tumors [3]. Our results indicated that the combination of Salmonella and cisplatin exerts additive therapeutic effects in delaying tumor growth and prolonging the survival of the tumor-bearing mice [3]. Recently, we further found that Salmonella not only cause increased intercellular communication between tumor cells due to $\mathrm{Cx} 43$ up-regulation, but also increased mitogen-activated protein kinase (MAPK) signaling pathways [33]. Salmonella presumably enhance the response to chemotherapy agents by increasing the passage of these drugs between neighboring tumor cells. The combination of Salmonella with 5-fluorouracil 
(5-FU) improved the antitumor efficacy compared with 5-FU monotherapy in pancreatic stem-like cells [34]. Chemotherapeutic agents that enhance antitumor efficacy would involve inhibiting the viable rim of tumor growth or modifying the tumor matrix, which would facilitate the motility and penetration of Salmonella [3]. By taking advantage of the tumoricidal effect of Salmonella and the pleiotropic activities of chemotherapeutic agents, Salmonella in combination with chemotherapy appears to hold promise for the treatment of solid tumors.

\section{Modification of Salmonella}

The anti-Salmonella immune responses that exist in the host influence the tumor-targeting potential of Salmonella after systemic administration. The loss of accumulation of Salmonella within the tumor contributes to the inhibition of the Salmonella-mediated antitumor response. The bacterial cell wall is negatively charged due to the presence of either teichoic acid in gram-positive bacteria or LPS in gram-negative bacteria. Positively charged polyelectrolytes can bond to negatively charged Salmonella surfaces to form thin films that circumvent preexisting immunity against Salmonella [35]. The encapsulation of Salmonella in polymer reduces the antigenicity of Salmonella following in vivo delivery. The polymer-modified Salmonella bacteria not only resist the binding of neutralizing antibodies in peripheral blood but also replicate in tumor sites, which is believed to stimulate non-specific antitumor immunity. In other words, polymer shields Salmonella from neutralizing antibodies. Polymer-modified Salmonella also display lower toxicity and improved efficacy and safety. Mice treated with Salmonella had a 9\% lower average body weight compared with mice treated with PBS. In contrast, the body weights of mice treated with polymer-modified Salmonella were not significantly decreased. After Salmonella and polymer-modified Salmonella treatment, the levels of inflammatory cytokines including interleukin-1 $\beta$ (IL-1 $\beta$ ) and tumor necrosis factor- $\alpha$ (TNF- $\alpha$ ) were measured in the sera. The induction of inflammation cytokines (i.e., IL-1 $\beta$ and TNF- $\alpha$ ) in mice treated with Salmonella was increased by 1.5-4.5-fold compared with induction by polymer-modified Salmonella treatment [35]. Polymer also can provide a useful platform for the chemical modification of Salmonella, perhaps allowing other chemotherapeutic drugs to bind to tumor-targeting Salmonella. Recently, the masking of Salmonella with a polymer and DNA resulted in the tumor-targeting gene transfer of Salmonella following in vivo delivery [36]. These results suggest that tumor-targeted gene therapy using polymer-modified Salmonella carrying the therapeutic gene, which exerts antitumor activities, represents a promising strategy for the treatment of tumors.

\section{Conclusions}

In this study, we have described the utility of Salmonella for tumor treatment. However, we believe that no powerful therapeutic agents are available to eradicate various types of tumor. Salmonella is a useful antitumor agent due to its tumor-targeting potential, antitumor capability, and ability to deliver therapeutic genes. Thus, Salmonella-mediated tumor therapy may have an important role in tumor treatment. These results will contribute to new research that gives thought to the complex interactions between bacteria and host immunity in order to maximize the chances of therapeutic success. 


\section{Acknowledgments}

This work was supported by grants from the National Science Council (NSC 101-2320-B-039-012-MY3; NSC 103-2815-C-039-045-B), China Medical University (CMU103-SR-45), and the Taiwan Ministry of Health and Welfare Clinical Trial and Research Center of Excellence (DOH102-TD-B-111-004).

\section{Author Contributions}

W.-W.C. and C.-H.L. wrote the manuscript.

\section{Conflicts of Interest}

The authors declare no conflict of interest.

\section{References}

1. Saga, K.; Kaneda, Y. Virosome presents multimodel tumor therapy without viral replication. Biomed. Res. Int. 2013, 2013, 764706.

2. Lee, C.H. Engineering bacteria toward tumor targeting for tumor treatment: Current state and perspectives. Appl. Microbiol. Biotechnol. 2012, 93, 517-523.

3. Lee, C.H.; Wu, C.L.; Tai, Y.S.; Shiau, A.L. Systemic administration of attenuated Salmonella choleraesuis in combination with cisplatin for tumor therapy. Mol. Ther. 2005, 11, 707-716.

4. Pawelek, J.M.; Low, K.B.; Bermudes, D. Bacteria as tumour-targeting vectors. Lancet Oncol. 2003, 4, 548-556.

5. Pawelek, J.M.; Low, K.B.; Bermudes, D. Tumor-targeted Salmonella as a novel antitumor vector. Cancer Res. 1997, 57, 4537-4544.

6. Liu, F.; Zhang, L.; Hoffman, R.M.; Zhao, M. Vessel destruction by tumor-targeting Salmonella typhimurium A1-R is enhanced by high tumor vascularity. Cell Cycle 2010, 9, 4518-4524.

7. Nagakura, C.; Hayashi, K.; Zhao, M.; Yamauchi, K.; Yamamoto, N.; Tsuchiya, H.; Tomita, K.; Bouvet, M.; Hoffman, R.M. Efficacy of a genetically-modified Salmonella typhimurium in an orthotopic human pancreatic tumor in nude mice. Antitumor Res. 2009, 29, 1873-1878.

8. Yam, C.; Zhao, M.; Hayashi, K.; Ma, H.; Kishimoto, H.; McElroy, M.; Bouvet, M.; Hoffman, R.M. Monotherapy with a tumor-targeting mutant of $S$. typhimurium inhibits liver metastasis in a mouse model of pancreatic tumor. J. Surg. Res. 2010, 164, 248-255.

9. Hayashi, K.; Zhao, M.; Yamauchi, K.; Yamamoto, N.; Tsuchiya, H.; Tomita, K.; Kishimoto, H.; Bouvet, M.; Hoffman, R.M. Systemic targeting of primary bone tumor and lung metastasis of high-grade osteosarcoma in nude mice with a tumor-selective strain of Salmonella typhimurium. Cell Cycle 2009, 8, 870-875.

10. Kimura, H.; Zhang, L.; Zhao, M.; Hayashi, K.; Tsuchiya, H.; Tomita, K.; Bouvet, M.; Wessels, J.; Hoffman, R.M. Targeted therapy of spinal cord glioma with a genetically modified Salmonella typhimurium. Cell Prolif. 2010, 43, 41-48. 
11. Sorenson, B.S.; Banton, K.L.; Frykman, N.L.; Leonard, A.S.; Saltzman, D.A. Attenuated Salmonella typhimurium with interleukin 2 gene prevents the establishment of pulmonary metastases in a model of osteosarcoma. J. Pediatr. Surg. 2008, 43, 1153-1158.

12. Lee, C.H.; Hsieh, J.L.; Wu, C.L.; Hsu, P.Y.; Shiau, A.L. T cell augments the antitumor activity of tumor-targeting Salmonella. Appl. Microbiol. Biotechnol. 2011, 90, 1381-1388.

13. Lee, C.H.; Wu, C.L.; Chen, S.H.; Shiau, A.L. Humoral immune responses inhibit the antitumor activities mediated by Salmonella enterica serovar Choleraesuis. J. Immunother. 2009, 32, 376-388.

14. Kaimala, S.; Mohamed, Y.A.; Nader, N.; Issac, J.; Elkord, E.; Chouaib, S.; Fernandez-Cabezudo, M.J.; Al-Ramadi, B.K. Salmonella-mediated tumor regression involves targeting of tumor myeloid suppressor cells causing a shift to M1-like phenotype and reduction in suppressive capacity. Cancer Immunol. Immunother. 2014, 63, 587-599.

15. Lee, C.H.; Wu, C.L.; Shiau, A.L. Toll-like receptor 4 mediates an antitumor host response induced by Salmonella choleraesuis. Clin. Cancer Res. 2008, 14, 1905-1912.

16. Kupz, A.; Curtiss, R., III; Bedoui, S.; Strugnell, R.A. In vivo IFN- $\gamma$ secretion by NK cells in response to Salmonella typhimurium requires NLRC4 inflammasomes. PLoS One 2014, 9, e97418.

17. Yoon, W.; Choi, J.H.; Kim, S.; Park, Y.K. Engineered Salmonella typhimurium expressing E7 fusion protein, derived from human papillomavirus, inhibits tumor growth in cervical tumor-bearing mice. Biotechnol. Lett. 2014, 36, 349-356.

18. Maybeno, M.; Redeker, A.; Welten, S.P.; Peters, B.; Loughhead, S.M.; Schoenberger, S.P.; Sette, A.; Arens, R. Polyfunctional $\mathrm{CD}^{+} \mathrm{T}$ cell responses to immunodominant epitopes correlate with disease activity of virulent Salmonella. PLoS One 2012, 7, e43481.

19. Saccheri, F.; Pozzi, C.; Avogadri, F.; Barozzi, S.; Faretta, M.; Fusi, P.; Rescigno, M. Bacteria-induced gap junctions in tumors favor antigen cross-presentation and antitumor immunity. Sci. Transl. Med. 2010, 2, 44ra57.

20. Avogadri, F.; Martinoli, C.; Petrovska, L.; Chiodoni, C.; Transidico, P.; Bronte, V.; Longhi, R.; Colombo, M.P.; Dougan, G.; Rescigno, M. Cancer immunotherapy based on killing of Salmonella-infected tumor cells. Cancer Res. 2005, 65, 3920-3927.

21. Manuel, E.R.; Blache, C.A.; Paquette, R.; Kaltcheva, T.I.; Ishizaki, H.; Ellenhorn, J.D.; Hensel, M.; Metelitsa, L.; Diamond, D.J. Enhancement of tumor vaccine therapy by systemic delivery of a tumor-targeting Salmonella-based STAT3 shRNA suppresses the growth of established melanoma tumors. Cancer Res. 2011, 71, 4183-4191.

22. Binder, D.C.; Engels, B.; Arina, A.; Yu, P.; Slauch, J.M.; Fu, Y.X.; Karrison, T.; Burnette, B.; Idel, C.; Zhao, M.; et al. Antigen-specific bacterial vaccine combined with anti-PD-L1 rescues dysfunctional endogenous T cells to reject long-established tumor. Cancer Immunol. Res. 2013, 1, 123-133.

23. Lee, C.H.; Hsieh, J.L.; Wu, C.L.; Hsu, H.C.; Shiau, A.L. B cells are required for tumor-targeting Salmonella in host. Appl. Microbiol. Biotechnol. 2011, 92, 1251-1260.

24. Reid, R.R.; Prodeus, A.P.; Khan, W.; Hsu, T.; Rosen, F.S.; Carroll, M.C. Endotoxin shock in antibody-deficient mice: Unraveling the role of natural antibody and complement in the clearance of lipopolysaccharide. J. Immunol. 1997, 159, 970-975. 
25. Toso, J.F.; Gill, V.J.; Hwu, P.; Marincola, F.M.; Restifo, N.P.; Schwartzentruber, D.J.; Sherry, R.M.; Topalian, S.L.; Yang, J.C.; Stock, F.; et al. Phase I study of the intravenous administration of attenuated Salmonella typhimurium to patients with metastatic melanoma. J. Clin. Oncol. 2002, $20,142-152$.

26. Ganai, S.; Arenas, R.B.; Sauer, J.P.; Bentley, B.; Forbes, N.S. In tumors Salmonella migrate away from vasculature toward the transition zone and induce apoptosis. Cancer Gene Ther. 2011, 18, 457-466.

27. Boise, L.H.; Collins, C.M. Salmonella-induced cell death: Apoptosis, necrosis or programmed cell death? Trends Microbiol. 2001, 9, 64-67.

28. Hernandez, L.D.; Pypaert, M.; Flavell, R.A.; Galán, J.E. A Salmonella protein causes macrophage cell death by inducing autophagy. J. Cell Biol. 2003, 163, 1123-1131.

29. Gomes, L.C.; Dikic, I. Autophagy in antimicrobial immunity. Mol. Cell 2014, 54, 224-233.

30. Lee, C.H.; Lin, S.T.; Liu, J.J.; Chang, W.W.; Hsieh, J.L.; Wang, W.K. Salmonella induce autophagy in melanoma by the down-regulation of AKT/mTOR pathway. Gene Ther. 2014, 21, 309-316.

31. Crull, K.; Weiss, S. Antibiotic control of tumor-colonizing Salmonella enterica serovar Typhimurium. Exp. Biol. Med. 2011, 236, 1282-1290.

32. Zhang, Y.; Zhang, N.; Su, S.; Hoffman, R.M.; Zhao, M. Salmonella typhimurium A1-R tumor targeting in immunocompetent mice is enhanced by a traditional Chinese medicine herbal mixture. Antitumor Res. 2013, 33, 1837-1843.

33. Chang, W.W.; Lai, C.H.; Chen, M.C.; Liu, C.F.; Kuan, Y.D.; Lin, S.T.; Lee, C.H. Salmonella enhance chemosensitivity in tumor through connexin 43 upregulation. Int. J. Cancer 2013, 133, 1926-1935.

34. Hiroshima, Y.; Zhao, M.; Zhang, Y.; Maawy, A.; Hassanein, M.K.; Uehara, F.; Miwa, S.; Yano, S.; Momiyama, M.; Suetsugu, A.; et al. Comparison of efficacy of Salmonella typhimurium A1-R and chemotherapy on stem-like and non-stem human pancreatic tumor cells. Cell Cycle 2013, 12, 2774-2780.

35. Lee, C.H.; Lin, Y.H.; Hsieh, J.L.; Chen, M.C.; Kuo, W.L. A polymer coating applied to Salmonella prevents the binding of Salmonella-specific antibodies. Int. J. Cancer 2013, 132, 717-725.

36. Wang, W.K.; Kuan, Y.D.; Kuo, C.Y.; Lee, C.H. Connexin 43 gene therapy delivered by polymer-modified Salmonella in murine tumor models. Polymers 2014, 6, 1119-1128.

(C) 2014 by the authors; licensee MDPI, Basel, Switzerland. This article is an open access article distributed under the terms and conditions of the Creative Commons Attribution license (http://creativecommons.org/licenses/by/3.0/). 